\title{
The elusive nature of white matter damage in anatomo-clinical correlations
}

\author{
Paolo Bartolomeo ${ }^{1,2 *}$ \\ INSERM, UMRS 975, Paris, France \\ 2 Department of Psychology, Catholic University, Milan, Italy \\ *Correspondence: paolo.bartolomeo@gmail.com
}

Edited by:

John J. Foxe, Albert Einstein College of Medicine, USA

Reviewed by:

Chris Rorden, Georgia Institute of Technology, USA

\section{A commentary on}

Is there a critical lesion site for unilateral spatial neglect? A meta-analysis using activation likelihood estimation

by Molenberghs, P., Sale, M. V., and Mattingley, J. B. (2012). Front. Hum. Neurosci. 6:78. doi: 10.3389/fnhum.2012.00078

Molenberghs et al. (2012) contributed a clearly written meta-analysis on the debated issue of the anatomy of spatial neglect. They looked for a critical lesion site for neglect, and found several distinct regions whose damage has been associated with signs of neglect. Lesioned clusters were located in virtually the whole lateral surface of the right hemisphere (see their Figure 1), as well as in the white matter.

Molenberghs et al.'s study is timely and much needed after the recent publication of new evidence concerning this debate (see, e.g., Saj et al., 2012). Despite a fairly complete covering of the literature, however, a general methodological point prevents studies such as the present one from giving adequate weight to lesions to long-range white matter pathways.

As the authors acknowledge in the general discussion, while their method based on "the peak coordinates of the critical lesion site" is certainly appropriate for gray matter lesions, it seems problematic for longrange white matter bundles. At variance with gray matter lesions, where one can look for maximum overlap (Vallar and Perani, 1986) or analogous topological data (Bates et al., 2003), lesions in different sectors along a long-range white matter fascicle can produce similar effects by disconnecting the fascicle, independent of the precise location of the interruption (Catani and Mesulam, 2008). This is a general problem for studies looking for a "critical lesion site" in braindamaged patients (Bartolomeo, 2011). Theoretically, similar behavioral deficits should be observed when a gray matter functional module is damaged as well as when white matter injury disconnects this module from the rest of the brain. Historically, neurologists have described neurological and neuropsychological deficits as disconnection syndromes (Geschwind, 1965; Catani and ffytche, 2005). However, the recent dominance of functional MRI (which identifies activation patterns in gray matter) and lesion symptom mapping based on gray matter injury (Bates et al., 2003) have led scholars to focus purely on the role of gray matter. Advances using techniques such as diffusion weighted imaging and the resulting tractography can help reveal the role of white matter (Catani, 2006). There is a clear need for methods that can integrate information from both gray and white matter injury, as these will likely provide better clinical significance and theoretical insight.

At present, the only way to explore the possibility that a deficit results from disconnection of a particular fascicle is to track the relevant fascicle, draw the lesions, and see whether or not they are located along the fascicle (see, e.g., Bourgeois et al., 2012). Methods based on this idea are being developed for group studies (Rudrauf et al., 2008). Concerning meta-analyses on neglect anatomy, Bartolomeo et al. (2007) mapped the hotspots defined by a small number of previous studies on fronto-parietal white matter, and found that the maximum lesion overlaps invariably occurred on or near the right superior longitudinal fasciculus (see also Doricchi et al., 2008; Thiebaut de Schotten et al., 2008). Such a procedure, however, would be much more cumbersome in larger meta-analyses such as the present one.
Despite these caveats, Molenberghs et al's results did reveal that "the largest region involved in the development of spatial neglect was a white matter lesion corresponding to the superior longitudinal fasciculus", consistent with the meta-analysis of Bartolomeo et al. (2007). However, other white matter sites of damage might have been more difficult to pinpoint. For example, the possibility that a disconnection of the inferior fronto-occipital fasciculus (IFOF) may determine neglect in some patients (Urbanski et al., 2008) does not appear in the meta-analysis results. This might well depend on the relative rarity of such patients; however, it could also result from the fact that the IFOF is a particularly long white matter pathway; thus, IFOFdamaging lesions might be dispersed along its length and go undetected by methods based on lesion clustering.

To conclude, Molenberghs et al. (2012) made an important attempt to clarify a complex problem such as the anatomy of neglect. Their results, however, also highlight demanding methodological issues that need to be solved in future research.

\section{REFERENCES}

Bartolomeo, P. (2011). The quest for the "critical lesion site" in cognitive deficits: problems and perspectives. Cortex 47, 1010-1012.

Bartolomeo, P., Thiebaut de Schotten, M., and Doricchi, F. (2007). Left unilateral neglect as a disconnection syndrome. Cereb. Cortex 45, 3127-3148.

Bates, E., Wilson, S. M., Saygin, A. P., Dick, F., Sereno, M. I., Knight, R. T., and Dronkers, N. F. (2003). Voxel-based lesion-symptom mapping. Nat. Neurosci. 6, 448-450. Bourgeois, A., Chica, A. B., Migliaccio, R., Thiebaut de Schotten, M., and Bartolomeo, P. (2012). Cortical control of inhibition of return: evidence from patients with inferior parietal damage and visual neglect. Neuropsychologia 50, 800-809.

Catani, M. (2006). Diffusion tensor magnetic resonance imaging tractography in cognitive disorders. Curr. Opin. Neurol. 19, 599-606. 
Catani, M., and ffytche, D. H. (2005). The rises and falls of disconnection syndromes. Brain 128, 2224-2239.

Catani, M., and Mesulam, M.-M. (2008). The arcuate fasciculus and the disconnection theme in language and aphasia: history and current state. Cortex 44 , 953-961.

Doricchi, F., Thiebaut de Schotten, M., Tomaiuolo, F., and Bartolomeo, P. (2008). White matter (dis)connections and gray matter (dys)functions in visual neglect: gaining insights into the brain networks of spatial awareness. Cortex 44, 983-995.

Geschwind, N. (1965). Disconnexion syndromes in animals and man - part I. Brain 88, 237-294.

Molenberghs, P., Sale, M. V., and Mattingley, J. B. (2012). Is there a critical lesion site for unilateral spatial neglect? A meta-analysis using activation likelihood estimation. Front. Hum. Neurosci. 6, 78. doi: 10.3389/ fnhum.2012.00078
Rudrauf, D., Mehta, S., and Grabowski, T. J. (2008). Disconnection's renaissance takes shape: formal incorporation in group-level lesion studies. Cortex 44, 1084-1096.

Saj, A., Verdon, V., Vocat, R., and Vuilleumier, P. (2012). "The anatomy underlying acute versus chronic spatial neglect" also depends on clinical tests. Brain 135, e207; author reply e208.

Thiebaut de Schotten, M., Kinkingnéhun, S. R., Delmaire, C., Lehéricy, S., Duffau, H., Thivard, L., Volle, E., Lévy, R., Dubois, B., and Bartolomeo, P. (2008). Visualization of disconnection syndromes in humans. Cortex 44, 1097-1103.

Urbanski, M., Thiebaut de Schotten, M., Rodrigo, S., Catani, M., Oppenheim, C., Touzé, E., Chokron, S., Méder, J.-F., Lévy, R., Dubois, B., and Bartolomeo, P. (2008). Brain networks of spatial awareness: evidence from diffusion ten- sor imaging tractography. J. Neurol. Neurosurg. Psychiatry 79, 598-601.

Vallar, G., and Perani, D. (1986). The anatomy of unilateral neglect after right-hemisphere stroke lesions. A clinical/CT-scan correlation study in man. Neuropsychologia 24, 609-622.

Received: 11 April 2012; accepted: 18 July 2012; published online: 03 August 2012.

Citation: Bartolomeo P (2012) The elusive nature of white matter damage in anatomo-clinical correlations. Front. Hum. Neurosci. 6:229. doi: 10.3389/fnhum.2012.00229 Copyright (C) 2012 Bartolomeo. This is an open-access article distributed under the terms of the Creative Commons Attribution License, which permits use, distribution, and reproduction in other forums, provided the original authors and source are credited and subject to any copyright notices concerning any third-party graphics etc. 